Tetrahedron Letters

journal homepage: www.elsevier.com

\title{
Photo-[4+4]-cycloaddition (para) of meta-substituted benzenes with 2-pyridones
}

\author{
Buddha B. Khatri ${ }^{\mathrm{a}}$, Darius Vrubliauskas ${ }^{\mathrm{a}}$ and Scott McN. Sieburth ${ }^{\mathrm{a}}{ }{ }^{\mathrm{a}}$ \\ ${ }^{a}$ Department of Chemistry, Temple University, 1901 N. $13^{\text {th }}$ Street, Philadelphia, Pennsylvania 19122 USA
}

ARTICLE INFO

Article history:

Received

Received in revised form

Accepted

Available online

Keywords:

Keyword_1

Keyword 2

Keyword_3

Keyword 4

Keyword_5

\section{ABSTRACT}

Benzene is unreactive as a photochemistry partner with 2-pyridone, however benzene substitution can allow for an efficient [4+4] (a k a, "para") photocycloaddition. Productive substituents include alkoxy, cyano, ester and trifluoromethyl, suggesting that inductively electron-withdrawing groups are sufficient, however a single fluorine substituent does not result in cycloaddition. When the product of this cycloaddition contains a conjugated system (e.g., an unsaturated ester) a secondary photocycloaddition can follow, forming triply bridged all-syn[3]ladderane products.

2009 Elsevier Ltd. All rights reserved.

* Corresponding author. Tel.: +1-215-204-7916; fax: +1-215-204-1532; e-mail: scott.sieburth@temple.edu 


\section{Introduction}

The photochemistry of benzene has been intensively studied for more than fifty years. ${ }^{1,2}$ In the presence of alkenes photocycloaddition can result, leading to ortho, meta and para adducts, e.g., $[2+2],[3+2]$ and [4+2] cycloadditions, Figure 1 . Meta cycloaddition of alkenes with benzene has been extensively exploited to construct a dazzling array of synthetic targets, ${ }^{3-5}$ while ortho and para cycloadditions of benzene have received less attention. ${ }^{5,6}$ Higher-order cycloadditions of benzene are even more rare. ${ }^{7}$ We report here the discovery that simple substitution of the benzene leads, in many cases, to para [4+4] photoreactivity with 2-pyridones.

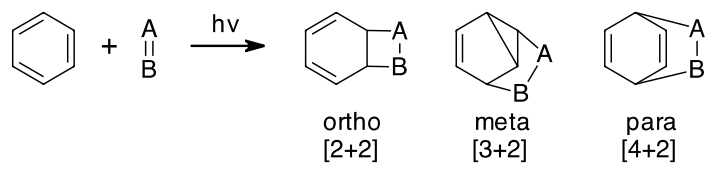

Figure 1. Three modes of benzene cycloaddition.

Pyridones are rather promiscuous [4+4] partners that undergo photocycloaddition with themselves, 1,3-dienes and several aromatics. $^{8} \quad$ For example, anthracene undergoes a rapid intramolecular cycloaddition with 2-pyridone give 2, Scheme $1 .{ }^{9}$ Similarly, a 1-naphthyl group yields a mixture of diastereomers 4 and 5. ${ }^{10}$ In contrast, a tethered benzene $(6)$ is inert to these conditions and dimers of the pyridone are the only observed products. ${ }^{10}$

The stability of benzene toward photocycloaddition with 2pyridones was not unexpected. We assumed that this lack of reactivity was a consequence of the stability of benzene relative to naphthalene and anthracene. ${ }^{9,10}$ Moreover, we and others ${ }^{11}$ have used benzene and toluene routinely as solvent for pyridone photochemistry. In one publication chlorobenzene, trifluoromethylbenzene and pyridine were all used as solvents without complication. ${ }^{12}$
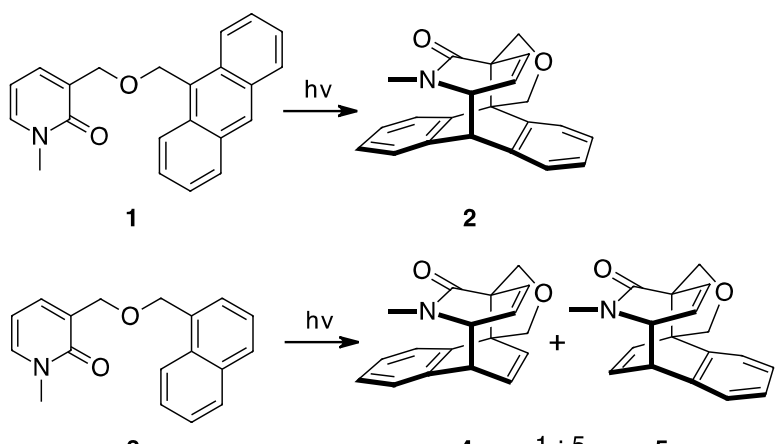

3

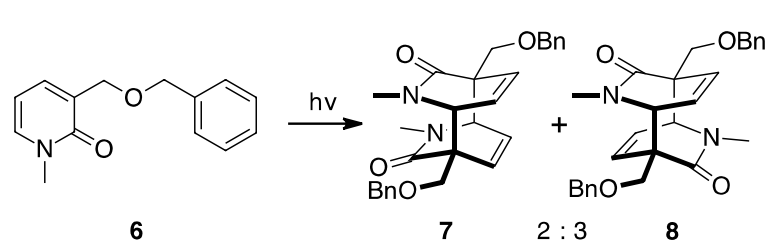

Scheme 1. Anthracene and naphthalene undergo rapid [4+4] photocycloaddition with 2-pyridones but benzene is inert. ${ }^{9,10}$

We have been intrigued by reports from Okumura and Gilbert describing the [4+4] photocycloaddition of benzonitrile with 2,3dimethyl-1,3-butadiene, a reaction that occurs with high regioselectivity - solely at the 2- and 5-positions of the ring - yielding 11, Scheme 2. ${ }^{13,14}$ Based on these reports, we elected to investigate nitrile-substituted benzene as a photocycloaddition partner with 2-pyridones.

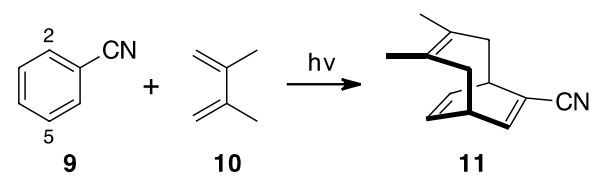

Scheme 2. Okumura and Gilbert's [4+4] photocycloaddition of benzonitrile. ${ }^{13,14}$

To test the effect of nitrile substitution, 12a was prepared and irradiated as a solution in benzene- $d_{6}$ (medium-pressure mercury lamp, Pyrex filtration ${ }^{15}$ ), Scheme 3. To our delight, photodimer 15a was not formed, and instead [4+4] cycloadduct 14a was the major adduct. We anticipated that diastereomer 13a would accompany 14a but it constituted less than $5 \%$ of the product mixture. The identity of $\mathbf{1 4 a}$ was easily ascertained because this strained product underwent a facile Cope rearrangement to give 17a. The nitrile of $\mathbf{1 7 a}$ resides at one end of a 1,3-diene, making it easily differentiated from the alternative 1,3-diene substitution of 16a that would result from Cope rearrangement of 13a. Structure 17a was also confirmed by X-ray crystallography (see Supporting Information). In addition to $\mathbf{1 4 a}$, we also isolated hexacyclic 18a. Compound 18a was clearly a secondary photoproduct, derived by [2+2] cycloaddition of the unsaturated nitrile of 14a and the nearby alkene. This secondary photocycloaddition was slower than the first, and the yield of 18a increased with increased irradiation time.

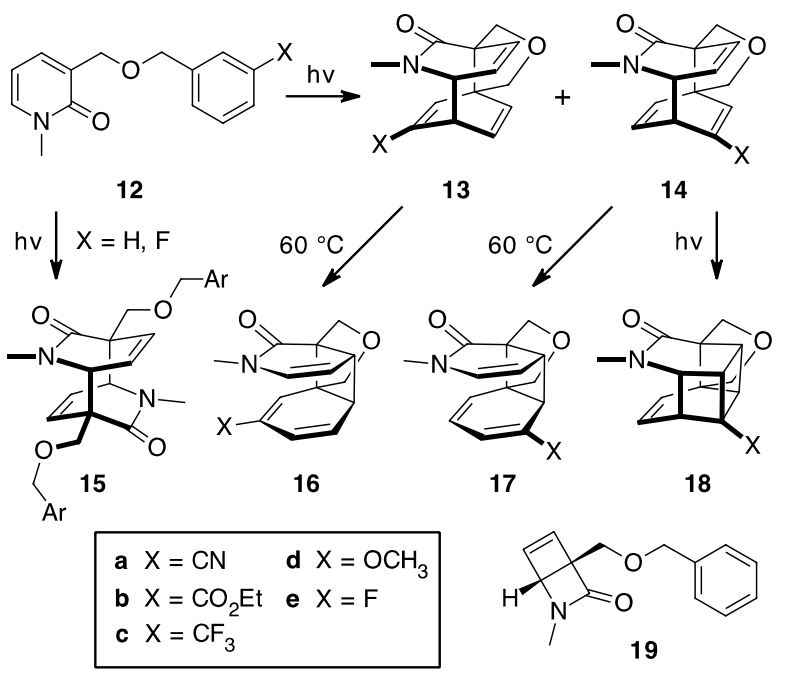

Scheme 3. Substitution of the benzene ring allows, in some cases, para-cycloaddition with 2-pyridone.

A nitrile substitutent is electron withdrawing through both induction and conjugation. To examine this effect more closely, we assembled a set of analogs, Scheme 3 and Table 1. Replacing the nitrile with an ester (12b) was expected to have an electronic effect on the benzene ring similar to the nitrile and possibly yield similar results. In the event, $\mathbf{1 2 b}$ it gave a mixture of $\mathbf{1 4 b}$ and $\mathbf{1 8 b}$, and negligible amounts of $\mathbf{1 3 b}$. In this case, the photoreaction of ester 12b was slower than the nitrile 12a and the secondary photoproduct $\mathbf{1 8 b}$ became a more prominent 
component of the mixture. The relatively slower cycloaddition between the 2-pyridone and the benzoate also allowed for formation of the other products, dimer $\mathbf{1 5 b}$ formation and isomerization product $\mathbf{1 9 b}$, Table 1 .

To probe the effect of having a purely inductively electronwithdrawing substitution, we tested trifluoromethyl analog $\mathbf{1 4 c}$. This substrate also underwent [4+4] cycloaddition, and like ester 14b this cycloaddition was slower than 14a resulting in a significant quantity of dimer $15 \mathbf{c}$ and isomerization product 19c. Unlike 14a and $\mathbf{1 4 b}$, the absence of a significant chromophore in product $\mathbf{1 4} \mathbf{c}$ resulted in photostability for this product.

With a meta-methoxy group, 12d, the substitutent is both inductively electron withdrawing and strongly electron donating through resonance. In this case, the cycloaddition was again para for the benzene and it was rapid, with little of the alternative photoproducts $15 d$ or 19d formed. For the first time, diastereomer 13d was a significant product component. Products 13d and 14d, like trifluoromethyl 14c, lacked a significant chromophore and were therefore photostable. Substrate 12d proved to be the highest yielding and the most facile of the cycloadditions.

The success of these inductively electron withdrawing substituents gave us confidence that the meta-fluoro 12e would also lead to benzene-pyridone cycloaddition. Nevertheless, this substrate only slowly underwent dimerization and isomerization to yield a mixture of $15 \mathrm{e}$ and $19 \mathrm{e}$.

Table 1. Substituted Benzene Photocycloadditions

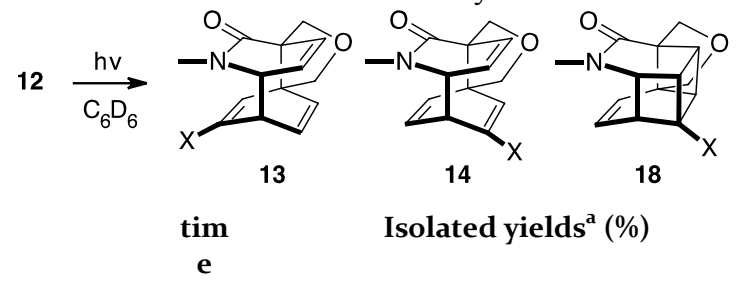

\begin{tabular}{|c|c|c|c|c|c|c|c|c|} 
& $\mathbf{X}$ & $(\mathbf{h})$ & $\mathbf{1 3}$ & $\mathbf{1 4}$ & $\mathbf{1 5}^{\mathbf{b}}$ & $\mathbf{1 8}$ & $19^{\mathrm{b}}$ & other \\
\hline $\mathbf{a}$ & $\mathrm{CN}$ & 4 & $<5$ & 35 & $<5$ & 20 & $<5$ & $13^{\mathrm{c}}$ \\
\hline $\mathbf{b}$ & $\mathrm{CO}_{2} \mathrm{Et}$ & 15 & $<5$ & 20 & 10 & 22 & 10 & - \\
\hline $\mathbf{c}$ & $\mathrm{CF}_{3}$ & 15 & $<5$ & 42 & 13 & - & 12 & - \\
\hline d & $\mathrm{OMe}$ & 1 & 21 & 46 & $<5$ & - & $<5$ & - \\
\hline e & $\mathrm{F}$ & 15 & $<5$ & $<5$ & $4 \mathrm{C}^{\mathrm{c}}$ & $<5$ & $10^{\mathrm{c}}$ & - \\
\hline
\end{tabular}

a. Yields reported for $\mathbf{1 3}$ and $\mathbf{1 4}$ are isolated yields of products 16 and 17, either after standing at $\mathrm{rt}$ or after warming the photoreaction mixture to $60{ }^{\circ} \mathrm{C}$ for several hours. b. intermolecular dimerization is a concentration-dependent reaction; the ratio of dimer $\mathbf{1 5}$ and isomerization product $\mathbf{1 9}$ is dependent on the initial concentration and the irradiation time. These yields are NMR estimates at ca. 50\% conversion after 15 h. c. tentatively assigned as an isomer of the higher-order metacycloaddition adduct $\mathbf{2 0}$.

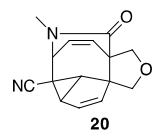

The strain inherent in aryl-aryl [4+4] cycloaddition adducts leads to relatively low temperature $[3,3]$ rearrangement of the 1,5-hexadiene embedded within these products, as has been noted previously. ${ }^{11,16}$ These Cope rearrangements can sometimes be observed at ambient temperature and also during chromatographic separation. For some of the products $\mathbf{1 3}$ and $\mathbf{1 4}$ formed here, these rearrangements made isolation of the primary photoproducts difficult. The mixtures were therefore warmed to induce full conversion to $\mathbf{1 6}$ and $\mathbf{1 7}$, which were then isolated. The yields reported in Table 1 for $\mathbf{1 3}$ and $\mathbf{1 4}$ are based on these isolated rearrangement products.

When the intramolecular photocycloadditions were slow (12b and 12c) or absent (12e), 2-pyridone dimerization and/or photoisomerization of the pyridone were observed. Pyridone photochemistry proceeds through a short-lived singlet excited state and the competition between the inter- and the intramolecular reaction paths is therefore dependent on the concentration of starting 12, which changes as the reactions progress. $^{11}$ The yields reported in Table 1 for $\mathbf{1 5}$ and 19 are expected, therefore, to be variable.

Higher-order photocycloaddition reactions of benzene and substituted benzenes have been reported sparingly, Scheme 4 . Butadiene and isoprene can add para to benzene to give products derived from $23 .{ }^{17}$ If this reaction is conducted in the presence of iodine, $\mathbf{2 3}$ is isomerized to $\mathbf{2 4}$, which can be isolated in low yield. ${ }^{18,19}$ The most extensive investigations of benzene higher order cycloadditions are the intramolecular cycloadditions of alkoxy-substituted benzenes with naphthalene and anthracene that yield products like $\mathbf{2 5}^{20,21}$

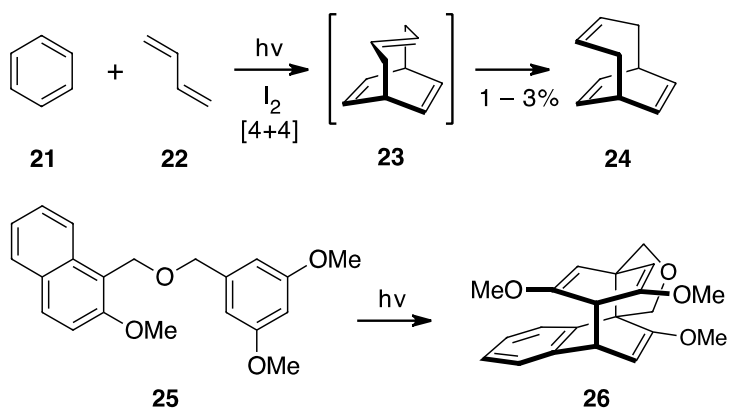

Scheme 4. Para photocycloaddition of benzenes with dienes, and aromatics.

The work by Gilbert (Scheme 2) utilized $254 \mathrm{~nm}$ lamps and wavelengths down to $220 \mathrm{~nm}$ (Vycor filter), whereas in this work light above $290 \mathrm{~nm}$ (Pyrex filter) was used. In these new examples, the light is presumably directed largely into the 2pyridone $\left(\lambda_{\operatorname{maz}}=305 \mathrm{~nm}\right)$ whereas of the tethered substituted benzenes have absorptions mostly below the Pyrex cutoff. The reported $\lambda_{\operatorname{maz}}$ for the substituted benzenes utilized here are in the range of 220-284 nm. ${ }^{22}$ UV spectra for the photosubstrates 13a-e used here can be found in the Supporting Information.

The photocycloadditions reported here raise numerous questions. The role of the substituent in facilitating photocycloaddition is not clear. Inspection of UV spectra for the substrates $\mathbf{1 3}$ and ${ }^{13} \mathrm{C}$ chemical shifts for the substituted benzenes do not provide insight. The stereoselectivity observed for several of the substrates, yielding trans isomers over cis products, may be electronic or steric. Many alternative substitutions such as ortho, para and even ipso attachment of the tether to C- 6 of the pyridone can be envisioned, as well as intermolecular studies. The use of pyridones in place of simpler 1,3-dienes results in more productive photochemistry for substituted benzenes and provide access to a variety of new structural motifs. All of these are 
currently under investigation and we expect to report these more wide ranging results in due course.

\section{Acknowledgments}

We gratefully acknowledge support from the National Science Foundation (1152159). The authors thank Professor Michael J. Zdilla and Dr. Shivaiah Vaddypally for the crystal structure of 17 a.

\section{References and notes}

1. Bryce-Smith, D.; Gilbert, A. Tetrahedron 1976, 32, 1309-1326

2. Bryce-Smith, D.; Gilbert, A. Tetrahedron 1977, 33, 2459-2489.

3. Wender, P. A.; Ternansky, R.; deLong, M.; Singh, S.; Olivera, A.; Rice, K. Pure. Appl. Chem. 1990, 62, 1597-1602.

4. Chappell, D.; Russell, A. T. Org. Biomol. Chem. 2006, 4, 44094430.

5. Streit, U.; Bochet, C. G. Beilstein. J. Org. Chem. 2011, 7, 525542.

6. Cornelisse, J.; de Haan, R. "Ortho Photocycloaddition of Alkenes and Alkynes to the Benzene Ring", in Understanding and Manipulating Excited-State Processes, Marcel Dekker: New York, $2001 ; 1-126$.

7. Norbert, H. "Ortho-, Meta-, and Para-Photocycloaddition of Arenes", in Synthetic Organic Photochemistry, Marcel Dekker: New York, 2005; 529-552.

8. Sieburth, S. McN. "Photochemical reactivity of pyridones", in CRC Handbook of Organic Photochemistry and Photobiology Horspool, W.; Lenci, F., Eds.; CRC Press: Boca Raton, FL, 2004; 103/1-103/18.

9. Khatri, B. B.; Kulyk, S.; Sieburth, S. McN. Org. Chem. Front. 2014, 1, 961-964.

10. Sieburth, S. McN.; McGee, K. F.; Zhang, F.; Chen, Y. J. Org Chem. 2000, 65, 1972-1977.

11. Nakamura, Y.; Kato, T.; Morita, Y. J. Chem. Soc,. Perkin. Trans. l 1982, 1187-1191.

12. Sieburth, S. McN.; McGee, K. F.; Al-Tel, T. H. J. Am. Chem. Soc. 1998, 120, 587-588.
13. Okumura, K.; Takamuku, S.; Sakurai, H. J. Soc. Chem. Ind. Japan 1969, 72, 200-203.

14. Gilbert, A.; Griffiths, O. J. Chem. Soc,. Perkin. Trans. 1 1993, 1379-1384.

15. Photoreactions were conducted with a $400 \mathrm{~W}$ medium-pressure mercury lamp housed in a water-cooled jacket with Pyrex ${ }^{\circledR}$ filtration. Solutions were prepared in benzene- $\mathrm{d}_{6}$, toluene or methanol, typically in $3.5 \mathrm{~mm}$ diameter NMR spectroscopy tubes and monitored by NMR and/or TLC. Alternatively,

photoreactions of similarly prepared samples were irradiated using a Southern New England Rayonet RPR-100 reactor equipped with $3000 \AA$ bulbs.

16. Sieburth, S. McN.; Lin, C. -H. J. Org. Chem. 1994, 59, $3597-$ 3599.

17. Kraft, K.; Koltzenburg, G. Tetrahedron Lett. 1967, 8, 4723-4728.

18. Löffler, H.-P. Tetrahedron Lett. 1974, 15, 787-788.

19. Carnadi, H. D.; Hildenbrand, P.; Richter, J.; Schröder, G. Eur. J. Org. Chem. 1978, 2074-2087.

20. Cao, D.; Dobis, S.; Meier, H. Tetrahedron Lett. 2002, 43, 68536855. Cao, D.; Dobis, S.; Schollmeyer, D.; Meier, H. Tetrahedron 2003, 59, 5323-5327. Dobis, S.; Schollmeyer, D.; Gao, C.; Cao, D.; Meier, H. Eur. J. Org. Chem. 2007, 2007, 2964 2969. Cao, D.; Gao, C.; Dobis, S.; Meier, H. Synthesis 2007, 2007, 1995-2001. Cao, D.; Meier, H. Tetrahedron Lett. 2005, 46, 4975-4977.

21. Cao, D.; Xu, S.; Gao, C.; Meier, H. Tetrahedron Lett. 2006, 47, $2759-2763$.

22. benzonitrile $\left(\lambda_{\operatorname{maz}}=221 \mathrm{~nm}\right)$ and anisole $\left(\lambda_{\operatorname{maz}}=220 \mathrm{~nm}\right)$, see: van Walree, C. A.; Roest, M. R.; Schuddeboom, W.; Jenneskens, L. W.; Verhoeven, J. W.; Warman, J. M.; Kooijman, H.; Spek, A. L. J. Am. Chem. Soc. 1996, 118, 8395-8407. Ethyl benzoate $\left(\lambda_{\operatorname{maz}}=\right.$ $284 \mathrm{~nm}$ ): Macanita, A. L.; Magalhaes, J.; Dias, A.; Teles, H.; Iglesias, E. J. Chem. Soc., Faraday. Trans. 1990, 86, 4011-4016. Trifluoromethylbenzene $\left(\lambda_{\operatorname{maz}}=253 \mathrm{~nm}\right)$ : Fontana, L. P.; Smith, H. E. J. Org. Chem. 1987, 52, 3386-3389.

\section{Graphical Abstract}

To create your abstract, type over the instructions in the template box below.

\section{Photo-[4+4]-cycloaddition (para) of meta- substituted benzenes with 2-pyridones}

Buddha B. Khatri, Darius Vrubliauskas, Scott McN. Sieburth

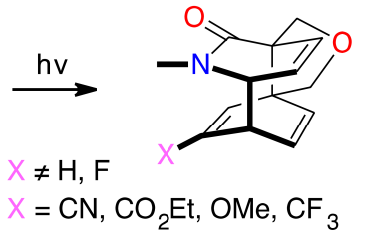

Leave this area blank for abstract info.
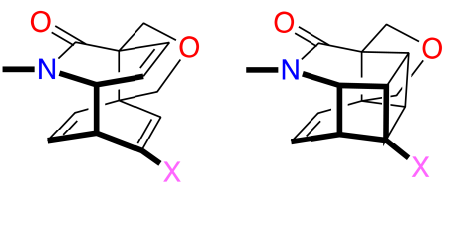

Fonts or abstract dimensions should not be changed or altered. 